\title{
New Eyes Looking at Solar Activity: Challenges for Theory and Simulations - Placing It into Context
}

\author{
S. Pohjolainen • M. Karlický • L. van Driel-Gesztelyi • \\ C.H. Mandrini
}

Published online: 25 November 2014

(C) The Author(s) 2014. This article is published with open access at Springerlink.com

\begin{abstract}
Solar Cycle 24 has opened a new era in solar radio physics as we now have instruments that can probe solar processes from submillimeter to kilometer waves. New and upgraded instruments provide data that enable studies of both energetic particles and thermal plasma, enhancing our knowledge of solar eruptions and acceleration and propagation of particles, through the solar chromosphere and corona and into the interplanetary space. In this Topical Issue we highlight the new observational capabilities and discuss the theoretical issues connected to solar radio emission and interplanetary radio physics.
\end{abstract}

\section{Introduction}

The solar atmosphere is a strong radio emission source, with the characteristic that shorter wavelengths probe deeper into the atmosphere and closer to the solar surface (Shibasaki,

New Eyes Looking at Solar Activity: Challenges for Theory and Simulations

Guest Editors: Silja Pohjolainen and Marian Karlický

S. Pohjolainen

Tuorla Observatory, University of Turku, Piikkiö, Finland

M. Karlický

Astronomical Institute of the Academy of Sciences of the Czech Republic, Ondrejov, Czech Republic

L. van Driel-Gesztelyi (

Observatoire de Paris, LESIA-CNRS UMR 8109, Meudon, France

e-mail: Lidia.VanDriel@obspm.fr

L. van Driel-Gesztelyi

Mullard Space Science Laboratory, University College London, Holmbury St. Mary, UK

L. van Driel-Gesztelyi

Konkoly Observatory of Hungarian Academy of Sciences, Budapest, Hungary

C.H. Mandrini

Instituto de Astronomía y Física del Espacio, CONICET-UBA, Buenos Aires, Argentina 
Alissandrakis, and Pohjolainen, 2011; Krucker et al., 2013). Solar transients like flares and coronal mass ejections can also generate strong radio emission (Pick and Vilmer, 2008). The wavelength range of solar emissions extends from submillimeter to kilometer waves, which imposes different kind of requirements on the radio telescopes and receivers, both ground-based and space-borne.

\section{Recent Development in Solar Radio Instrumentation}

The newly constructed Atacama Large Millimeter/submillimeter Array (ALMA) is one of the largest ground-based astronomy projects of the decade. When completed, ALMA will be comprised of an array of 66 antennas located in the Chilean Andes. The operational frequencies, from 950 to $30 \mathrm{GHz}$, will provide both linear and circular polarization that will probe into the lower, dense layers of the solar atmosphere. In 2014 the observations are in verification phase and proposals for solar observing time are under way. Being an astronomy facility, ALMA is not solar-dedicated, and it will provide only limited solar observing time. Thus, observations will be mostly focused on quiet Sun phenomena because transient and localized processes like flares are always more difficult to observe. We expect that solar ALMA observations will bring more information on the chromospheric thermal structure and dynamics, including waves, shocks, and heating (Benz et al., 2012).

The ALMA Regional Centres (ARCs) provide the interface between ALMA and scientists. The ARC node specialized in solar data analysis is in Ondřejov, Czech Republic. It provides help in observation planning, data quality check, data storage and processing, and data reduction using CASA (Karlický et al., 2011).

The Expanded Owens Valley Solar Array (EOVSA) project, to be completed soon, is expanding the array from the current seven antennas to a total of 15 antennas. In the upgrade the existing control systems, wiring, and signal processing will be replaced by modern ones, based on new technology. The new and longer baselines will provide more detailed imaging of solar active regions at a wide frequency range, from 18 to $1 \mathrm{GHz}$. The science to be addressed focuses on the magnetic structure of the solar corona, on transient phenomena resulting from magnetic interactions, and on space weather phenomena.

The recently upgraded and renamed Karl G. Jansky Very Large Array (VLA) has expanded its technical capacities as old electronics have been replaced with state-of-the-art equipment. The first solar observations by the upgraded VLA were made in 2011 in the $2-1 \mathrm{GHz}$ frequency band. Type III radio bursts at decimeter wavelengths were imaged with high time and frequency resolution, allowing electron beam trajectories in the corona to be deduced (Chen et al., 2013).

The requirement of a new instrument that is capable of wide frequency-range spectral imaging with high temporal, spatial, and spectral resolution led to the development of the Chinese Spectral Radioheliograph (CSRH). The Chinese solar physics community had planned to build a radioheliograph since the 1960s and after prototype testing in the early 2000s the construction of CSRH started in 2009. In 2014, first images of the quiet Sun have been obtained and test observations are under way. The frequency range of the instrument will be $15 \mathrm{GHz}-400 \mathrm{MHz}$ (Yan et al., 2009). The CSRH frequency range will complement the existing, upgraded Nancay Radioheliograph (NRH) that operates in the $500-150 \mathrm{MHz}$ frequency range in France, and the Nobeyama Radioheliograph (NoRH) that will continue its operation at 17 and $34 \mathrm{GHz}$ in Japan, under the International Consortium for the Continued Operation of Nobeyama Radioheliograph (ICCON) agreement in 2015.

Recently, the first solar observations using the Low-Frequency Array (LOFAR), an interferometric array of thousands of small antennas that operate in the frequency range of 
$240-10 \mathrm{MHz}$, have revealed multiple type III bursts several hours after the launch of two coronal mass ejections (Morosan et al., 2014). The low intensity bursts were detected due to LOFAR's superb sensitivity and angular resolution, which are estimated to be at least one order of magnitude better compared to those of older radio telescopes.

Several other upgrades and installations of new equipment have been carried out in the recent years. Among these are the Siberian Solar Radio Telescope (SSRT) operating in Irkutsk, Russia. The cross-shaped interferometer observes solar activity in the microwave range, at $5.7 \mathrm{GHz}$. The e-Callisto spectrometers (Monstein, 2013) have now been installed at dozens of locations all over the world. The simple antenna structures and cost-effective receiver technology have made it possible to monitor solar activity 24 hours a day at decimetermeter waves, in the $870-45 \mathrm{MHz}$ frequency range.

In space, the Wind WAVES and the Solar Terrestrial Relations Observatory (STEREO) SWAVES experiments remain in operation, providing continuous low frequency radio data in decameter-kilometer waves that cannot be recorded at Earth. The cut-off frequency for ground-based observations is the frequency below which radio waves fail to penetrate the Earth's ionosphere. The satellite data can be used to study plasma emission caused by solar transients, as well as other space weather effects.

\section{Topics in This Issue}

In this Topical Issue, Mendoza-Torres et al. (2015) describe the results from test observations with a 5-m radio telescope that uses novel planar feeds, designed to detect circularly polarized emission in microwaves. Planar feeds are based on microstrip technology, which is cheap, light, and simple for manufacturing.

On the quiet Sun emission, the observations of small-scale structures using the Radio Astronomical Telescope of the Academy of Sciences 600 (RATAN-600) spectral-polarization facility have shown that the microwave emission comes from a region that extends from the chromosphere to the low transition region, and it includes various structures like supergranulation network, bright points, and plage patches (Bogod et al., 2015). The NoRH and SSRT observations of solar active regions have also lead to the identification of long-lived intersunspot sources (ISSs). Two different types were discovered by Bakunina et al. (2015) and a qualitative three-flux model was proposed to explain the existence of long-lasting ISSs. Ryabov et al. (2014) studied large isolated sunspot regions and found a correspondence between the locations of the depressed microwave emission and the reduced soft X-ray emission. Their model estimates showed that a decrement of plasma density in both the coronal and lower layers above the microwave depression region can account for the depression, which they attributed to outward plasma flows from these regions.

The results from the first observations of sporadic radio emission at decameter wavelengths by the Giant Ukrainian Radio Telescope (GURT) are presented and discussed by Dorovskyy et al. (2015). The wide frequency range of the instrument facilitates the registration of harmonic pairs and a U-burst showing harmonic emission is analyzed in detail. Melnik et al. (2015) used simultaneous data obtained from several radio telescopes to observe those solar radio type III bursts that change the sign of their drift rate. Their obtained basic explanation differs from the common assumption that positive drift rates of type III bursts are connected with electron beam propagation toward the Sun. These authors propose that positive drift rates can also be created by outward moving electrons in coronal regions where the group velocities of type III emission are lower than the speed of the electron beam.

The Torun radio spectrograph data, with a high time-resolution of 80 microseconds, were used to study the time evolution of power spectra and Fourier spectral indices in selected 
short radio bursts (Dabrowski, Karlický, and Rudawy, 2015). The results suggest that dmspikes and drifting pulsation structure (DPS) events are physically similar and that both are signatures of cascades of interacting plasmoids of different sizes. A statistical analysis of spectral and temporal fine-structures of microwave emission during solar flares was carried out by Zhdanov and Zandanov (2015). The observations were obtained by the Badary Broadband Microwave Spectropolarimeter (BBMS) and compared with the SSRT interferometer data. The fine-structures of radio bursts observed by the Solar Broadband Radio Spectrometer (SBRS) at Huairou and the IZMIRAN spectrograph were studied in detail by Chernov et al. (2015). These observations help in testing the different theoretical models of fine-structure formation, as, for example, several mechanisms have been proposed for just the generation of zebra structures. Stanislavsky et al. (2015) have further used the observed zebra patterns and a band-split radio type II burst to determine the magnetic-field strengths in the corona.

A catalog of metric type IV radio bursts observed with the ARTEMIS-IV solar radio spectrograph was compiled by Bouratzis et al. (2015). As type IV bursts can be associated with both flare emissions and coronal mass ejection (CME) events, they provide valuable information on the energy release processes. The observed fine-structures in the cataloged events were classified and compared with the X-ray and microwave data and the associated CME onset times. The results indicate a very tight temporal association between energy release episodes and pulsations, spikes, narrow-band type III bursts, and zebra bursts. The possibility of filament eruptions to be responsible for shocks that propagate ahead of CMEs like decelerating blast waves was studied by Grechnev et al. (2015). The two analyzed events show shocks associated with a fast CME (where the shock turns into a bow shock) and a slow CME (a decaying shock). Radio type I noise storms are the most common phenomenon observed at metric wavelengths but they are generally not linked to flares or other coronal transients. Sodré, Cunha-Silva, and Fernandes (2015) have analyzed a large data set of type I chains, recorded with one of the spectrographs integrated in the e-Callisto network, and determined their physical parameters.

The pre-flare phases of active regions were investigated by Abramov-Maximov et al. (2015), to reveal features in the microwave radiation and in the magnetic-field characteristics that could indicate that a powerful flare is about to take place. They discovered that an early detection of a rapidly developing, neutral line associated microwave source and an increasing magnetic-field gradient can be used as a factor in predicting large flares. Szaforz and Tomczak (2015) tested the model of oscillating magnetic traps with the hard X-ray light curves observed by the Yohkoh satellite. They conclude that the analyzed solar flare data are consistent with the predictions of the model on how quasi-periodic pulsations (QPPs) are excited. Kuznetsov and Kontar (2015) used the recently developed interactive tool, GX Simulator, to simulate the gyrosynchrotron microwave emission in a flare. The comparison to multi-wavelength observations allowed determination of the spatial and spectral properties of the non-thermal electron distribution in the flaring loop.

The capabilities and results obtained with these instruments, and many more, were discussed widely during the CESRA Workshop, held in Prague, Czech Republic, in June 2013. The workshop took place under the kind auspices of Lord Mayor of the City of Prague, whose warm hospitality we wish to thank. The local organizers, led by Dr. Miroslav Bárta, made the meeting run smoothly and efficiently, to the benefit of all the participants.

Open Access This article is distributed under the terms of the Creative Commons Attribution License which permits any use, distribution, and reproduction in any medium, provided the original author(s) and the source are credited. 


\section{References}

Abramov-Maximov, V.E., Borovik, V.N., Opeikina, L.V., Tlatov, A.G.: 2015, Solar Phys. DOI, this issue. Bakunina, I.A., Melnikov, V.F., Solovev, A.A., Abramov-Maximov, V.E.: 2015, Solar Phys. DOI, this issue.

Benz, A.O., Brasja, R., Shimojo, M., Karlický, M., Testi, L.: 2012, Proc. IAU Special Session 6, Science with Large Solar Telescopes, id. E2.05.

Bogod, V.M., Alissandrakis, C.E., Kaltman, T.I., Tokhchukova, S.K.: 2015, Solar Phys. DOI, this issue.

Bouratzis, C., Hillaris, A., Alissandrakis, C.E., Preka-Papadema, P., Moussas, X., Caroubalos, C., Tsitsipis, P., Kontogeorgos, A.: 2015, Solar Phys. DOI, this issue.

Chen, B., Bastian, T.S., White, S.M., Gary, D.E., Perley, R., Rupen, M., Carlson, B.: 2013, Astrophys. J. Lett. 763, L21.

Chernov, G., Fomichev, V., Tan, B., Yan, Y., Tan, C., Fu, Q.: 2015, Solar Phys. DOI, this issue.

Dabrowski, B.P., Karlický, M., Rudawy, P.: 2015, Solar Phys. DOI, this issue.

Dorovskyy, V.V., Melnik, V.N., Konovalenko, A.A., Bubnov, I.N., Gridin, A.A., Shevchuk, N.V., et al.: 2015, Solar Phys. DOI, this issue.

Grechnev, V.V., Uralov, A.M., Kuzmenko, I.V., Kochanov, A.A., Chertok, I.M., Kalashnikov, S.S.: 2015, Solar Phys. DOI, this issue.

Karlický, M., Bárta, M., Dabrowski, B.P., Heinzel, P.: 2011, Solar Phys. 268, 165.

Krucker, S., Gimenez de Castro, C.G., Hudson, H.S., Trottet, G., Bastian, T.S., Hales, A.S., et al.: 2013, Astron. Astrophys. Rev. 21, 58.

Kuznetsov, A.A., Kontar, E.P.: 2015, Solar Phys. DOI, this issue.

Melnik, V.N., Brazhenko, A.I., Konovalenko, A.A., Briand, C., Dorovskyy, V.V., Zarka, P., et al.: 2015, Solar Phys. DOI, this issue.

Mendoza-Torres, J.E., Colin-Beltran, E., Corona-Chavez, A., Palacios-Fonseca, J.S., Rodriguez-Pedroza, B., Tlatelpa-Osorio, Y.E., et al.: 2015, Solar Phys. DOI, this issue.

Monstein, C.: 2013, EGU General Assembly, id. EGU2013-2027.

Morosan, D.E., Gallagher, P.T., Zucca, P., Fallows, R., Carley, E.P., Mann, G., et al.: 2014, Astron. Astrophys. 568, A67.

Pick, M., Vilmer, N.: 2008, Astron. Astrophys. Rev. 16, 1.

Ryabov, B.I., Gary, D.E., Peterova, N.G., Shibasaki, K., Topchilo, N.A.: 2014, Solar Phys. DOI, this issue.

Shibasaki, K., Alissandrakis, C.E., Pohjolainen, S.: 2011, Solar Phys. 273, 309.

Sodré, Z.A.L., Cunha-Silva, R.D., Fernandes, F.C.R.: 2015, Solar Phys. DOI, this issue.

Stanislavsky, A.A., Konovalenko, A.A., Koval, A.A., Dorovskyy, V.V., Zarka, P.H., Rucker, H.O.: 2015, Solar Phys. DOI, this issue.

Szaforz, Z., Tomczak, M.: 2015, Solar Phys. DOI, this issue.

Yan, Y., Zhang, J., Wang, W., Liu, F., Chen, Z., Ji, G.: 2009, Earth Moon Planets 104, 97.

Zhdanov, D.A., Zandanov, V.G.: 2015, Solar Phys. DOI, this issue. 